Ann. Biol. anim. Bioch. Biophys., 1977, 17 (5 B), 775-786.

\title{
Etude morphologique du tissu adipeux de couverture au stade de sa formation chez le fotus de porc
}

\author{
par N. VODOVAR, Françoise DESNOYERS, M. ETIENNE*
}

avec la collaboration technique de S. DELPAL ef G. PAILLARD

Station de Recherches de Nutrition,

* Station de Recherches sur l'Elevage des Porcs, I.N.R.A., 78350 Jouy-en-Josas.

Summary. Morphological study of pig fetus subcutaneous adipose tissue in the formative stage.

Development of subcutaneous adipose tissue in Large-White swine was studied. The first lipogenic cells in the various sites of tegument at inguinal, dorsal, scapular, sternal and neck levels appeared between days 52 and 56 of pregnancy.

In the subcutaneous adipose tissue area two layers separated by a dense connective tissue barrier were observed before and at the beginning of lipogenesis.

Components of connective, vascular and nervous tissues were distributed within each layer ; the aspect of these differed according to site of tissue sampling.

The first multivacuolar adipose cells of endothelial type were observed near developing capillaries.

Small and isolated lipid droplets were occasionally seen in cells of the fibroblastic type ; those cells, however, never became adipocytes.

Before and during the occurrence of lipid droplets, the aspect of preadipocytes was polymorphous and variable according to the sampling site. About ten days after the onset of lipogenesis, the mean size of adipose cells differed with the sampling site.

\section{Introduction.}

La morphologie structurale et ultrastructurale des tissus adipeux de Porc a été étudiée au cours de ces dernières années en tenant compte de l'âge de l'animal ef de la localisation anatomique de ces tissus dans l'organisme.

Pour les tissus adipeux internes de cette espèce et suivant leur emplacement, un certain nombre de travaux ont été fạits d'une part chez l'animal au stade fœetal (Vodovar, Desnoyers et François, 1971 ; Desnoyers, 1973 ; Hermans, 1973 ; Desnoyers et Vodovar, 1974 ; Desnoyers et Vodovar, 1977) ef d'autre part pendant la période de croissance de l'animal (Vodovar, Serres et François, 1969 ; Vodovar, Desnoyers ef Flanzy, 1972 ; Desnoyers, 1973). 
Le tissus adipeux de couverture a été peu étudié du point de vue morphologique; le récent travail de Mersmann, Goodman et Brown (1975) concerne la période postnatale et de rares indications ont été fournies concernant l'apparition de ce tissu (Alexandrowicz, 1949 ; Fowler et Calhoun, 1964).

Ełant donné l'importance qu'on attribue actuellement au tout premier stade du développement des tissus adipeux, quelle que soit l'espèce étudiée et leur localisation anatomique (Knittle ef Hirsch, 1968 ; Lemonnier, 1972 ; Lee, Kauffman et Grummer, 1973, parmi d'autres) et du fait que les tissus adipeux de couverture particulièrement bien développés chez le Porc, n'ont pas été étudiés avant la naissance, nous avons entrepris leur investigation. Nous présentons ici les observations concernant leur apparition et leur formation. Dans le texte le terme « lipogenèse » est employé pour indiquer l'apporition des lipides dans les cellules sans préjuger de leur provenance.

L'aspect morphologique du tégument, qui est très complexe et variable suivant les niveaux, a été étudié chez le fotus à différents âges (Fowler et Calhoun, 1964) et à la naissance du porcelet (Smith et Calhoun, 1964). Nous ne reviendrons donc pas sur ces descriptions, la région dans laquelle apparaît et se développe le tissu adipeux, faisant seul l'objet de ce travail.

\section{Matériel ef méthodes.}

Pour déterminer le moment d'apparition de la lipogenèse dans le tégument du foetus de Porc, des observations préliminaires et échelonnées dans le temps ont été faites à partir du $30^{\mathrm{e}}$ jour de la gestation aux niveaux inguinal, dorsal, interscapulaire, du cou et du sternum,. Les premiers foyers de lipogenèse ont été observés au niveau inguinol chez le fœtus âgé de 52 jours.

Les fœtus de Porc ont été prélevés sur des truies de race Large White, en bonne santé et recevant un régime équilibré. Les prélèvements ont été pratiqués tous les jours entre le $50^{\mathrm{e}}$ ef le $60^{\mathrm{e}}$ jour de la gestation et tous les deux jours entre le $60^{\mathrm{e}}$ et le 80 e jour. Les prélèvements des fœetus ont été réalisés soit au cours d'opération sur les truies, soit après leur abattage, au moyen des techniques exposées (Desnoyers, 1973 ;

\section{PLANCHE I}

Coupes semi-fines de téguments de fœłus de Porc au niveau interscapulaire

FIG. 1. - Vue d'une portion de coupe, perpendiculaire à la surface du tégument au niveau interscapulaire, chez un faetus de parc âgé de 50 jours. Aspect de la lame de tissu conjonctif, LC, et d'une partie de la « couche externe », CE, ef de la « couche interne», Cl. $\times 450$.

FIG. 2. - Au même niveau que celui de la figure 1, chez un foefus âgé de 52 jours, on note la présence des premières cellules adipeuses, CA, dans la couche externe, CE, et dans la couche interne, $\mathrm{Cl} . \times 450$.

FIG. 3. - Aspect d'un panicule adipeux observé dans la couche externe prélevé à un niveau correspondant à celui des figures 1 ef 2 , chez un foelus âgé de 78 jours. Les cellules adipeuses, CA, sont groupées autour des capillaires sanguins, $C . \times 450$.

FIG. 4. - Aspect d'un panicule adipeux observé dans la couche inferne prélevé chez le même fœius que celui de la figure 3 et au même niveau. Les cellules adipeuses, CA, sont plus volumineuses, tandis que leur nombre par panicule reste comparable. $\nsucc .450$. 
PI. I
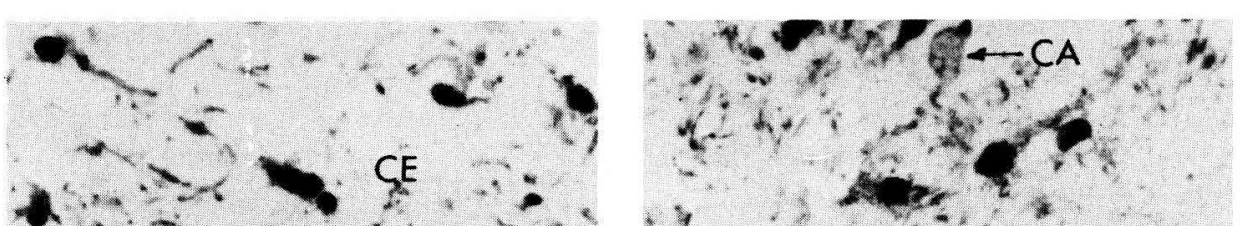

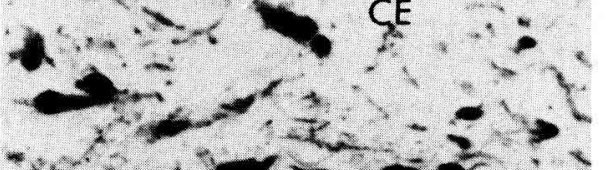

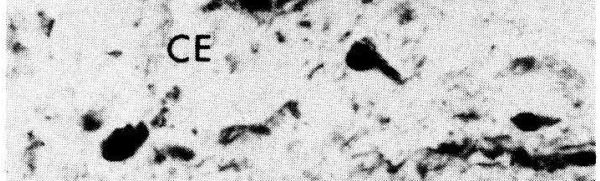

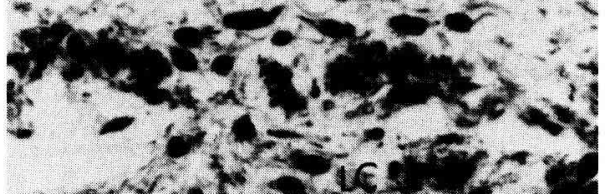

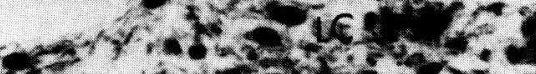

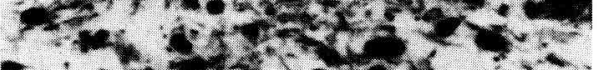
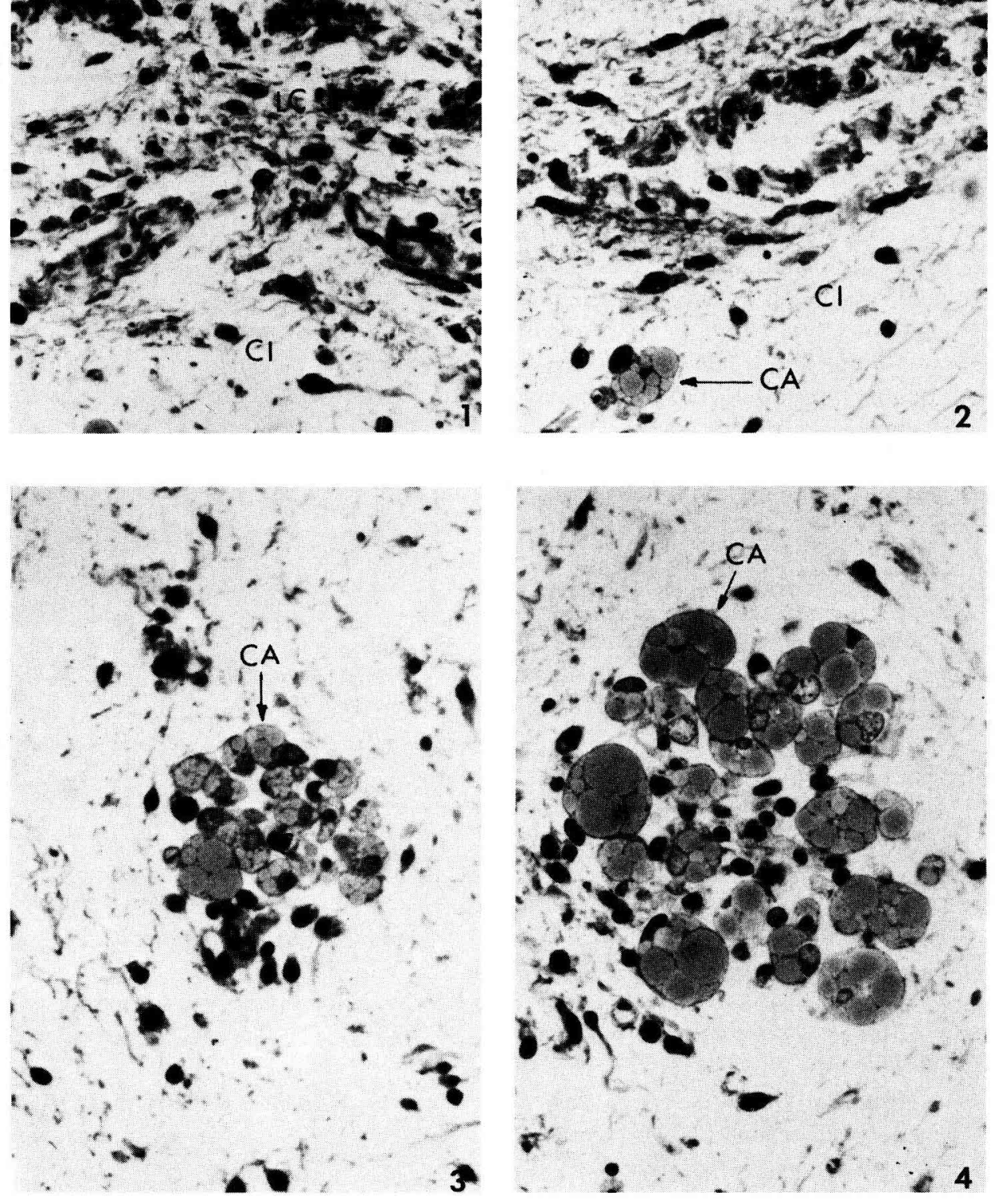
Desnoyers et Vodovar, 1974) et en tenant comple du nombre de fœtus par portée, de leur position dans l'utérus et de leur poids.

Le prélèvement des tissus, leur fixation ef leur préparation pour la microscopie photonique et la microscopie électronique ont été décrits précédemment (Vodovar, Desnoyers et François, 1971).

\section{Observations.}

1. Apparition de la lipogenèse dans le tégument du fœtus du Porc.

Sur les coupes en congélation, perpendiculaires à la surface du tégument, des tissus prélevés sur les fœtus de Porc âgés de 30, 40, 50 ef 51 jours aux niveaux inguinal, dorsal, interscapulaire, du cou et du sternum, il n'a pas été observé de cellules avec des inclusions lipidiques ni à l'examen direct ni après immersion dans le tétro. xyde d'osmium ou dans le Noir Soudan B.

Les premières cellules avec des inclusions osmiophiles et soudanophiles ont été observées au niveau inguinal chez le fœtus de Porc âgé de 52 jours. Pour l'ensemble des fœius et suivant les localisations citées ci-dessus, la lipogenèse apparaît entre le $52^{\mathrm{e}}$ et le $56^{\mathrm{e}}$ jour de la gestation. Pour la majorité des fotus, l'apparition de la lipogénèse semble se faire simulianément aux cinq niveaux de prélèvement tandis que pour quelques fœetus elle paraît s'échelonner, suivant le niveau, dans un laps de temps ne dépassant pas trois jours. Dans ce dernier cas, c'est toujours au niveau inguinal qu'on observe les premiers foyers de lipogenèse. Le nombre de fœus por portée, leur poids et leur emplacement dans la corne utérine ne semble pas influencer le moment d'apparition de la lipogenèse.

\section{Aspect en microscopie photonique.}

Sur les coupes semi-fines de tégument prélevé aux niveaux indiqués précédemmenł sur les fœetus de 50 jours, soit quelques jours avant l'apparition de la lipogenèse, on note pour les cinq localisation étudiées, que la région où les cellules adipeuses apparaîtront est constituée par deux couches d'aspect comparable séparées par une lame de tissu conjonctif (fig. 1).

L'épaisseur de chacune des deux couches, que nous appelons « couche externe» ef « couche interne » suivant leur situation par rapport à la lame conjonctive est variable pour un même niveau et pour une même couche suivant les niveaux des prélèvements. La population cellulaire disséminée dans ces deux couches est apparemment uniforme et seule une trame de tissu conjonctif assure la liaison entre les cellules. Les vaisseaux sanguins, généralement entourés d'un nombre réduit ef variable de cellules, et les éléments nerveux sont aussi disséminés et peu abondants. La lame conjonctive est compacte, mais son aspect est plus ou moins différent suivant le niveau du prélèvement.

L'apparition de la lipogenèse, qui peut varier chez certains fœtus dans la limite de quelques jours suivant le niveau du prélèvement, semble être, pour un niveau donné, toujours simultanée dans la couche externe et dans la couche interne.

Les premières cellules adipeuses (fig. 2) sont en contact ou à proximité immédiate 
des vaisseaux sanguins, en parficulier dans la couche inferne. Ces cellules, dont le nombre des inclusions lipidiques est toujours important, ont une forme plus ou moins ovale ou sphérique suivant l'incidence de la coupe. Leur volume, qui est surtout fonction des lipides accumulés, est variable ; cependant, le diamètre des cellules adipeuses les plus évoluées est, à cet âge, inférieur à $18 \mu \mathrm{m}$ si la forme cellulaire est assimilée d̀ une sphère.

Dans les jours qui suivent l'apparition des premières cellules adipeuses, on observe que, progressivement, les autres cellules groupées autour des capillaires sanguins se remplissent de lipides formant ainsi de véritables panicules de tissu adipeux lorsque le fœtus est âgé de 70 à 75 jours. Ces petits panicules, disséminés dans les deux couches, sont formés d'un nombre apparemment comparable de cellules adipeuses toujours associées à un ou plusieurs vaisseaux et parfois à quelques cellules sans lipides. Les éléments du tissu conjonctif préexistant sont repoussés à la périphérie de chaque groupe de cellules.

L'aspect de la répartition topographique des panicules adipeux à l'intérieur de chacune des deux couches est semblable au même niveau. Il en est de même pour la disposition des cellules adipeuses autour des vaisseaux sanguins à condition que l'incidence des coupes par rapport à l'axe du vaisseau soit comparable.

En ce qui concerne la taille des cellules adipeuses des deux couches, à un niveau donné, on constate que les cellules de la couche externe ont toujours un diamètre moyen inférieur à celui des cellules de la couche interne (fig. 3 et 4). Les cellules adipeuses, qui sont présumées apparaître au même moment, ont un diamètre moyen qui diffère suivant le niveau du prélèvement que ce soit dans la couche externe ou dans la couche interne. Chez le fœitus âgé de 75 jours et dans nos conditions d'expérience, le diamètre moyen des cellules adipeuses de la couche interne est de $16 \mu \mathrm{m}$ au niveau inguinal et de $13 \mu \mathrm{m}$ au niveau du cou. Entre les valeurs moyennes de ces deux localisations et par ordre décroissant se place les cellules des niveaux sternal, interscapulaire ef dorsal.

\section{Aspect en microscopie électronique.}

Observées environ $24 \mathrm{~h}$ avant l'apparition de la lipogenèse puis pendant celle-ci, les cellules, à l'emplacement des tissus adipeux de couverture et suivant leur répartition topographique, ont des caractéristiques morphologiques différentes. Mis à part les éléments du système nerveux ef du système vasculaire, on constate que la population cellulaire disséminée dans les deux couches décrites dans le paragraphe précédent, est de type fibroblastique (fig. 5), tandis que les cellules groupées autour des capillaires sont de type endothélial.

- Les cellules du premier type sont fusiformes, n'ont pas de lame basale et leur membrane plastique, à de rares exceptions près, est régulière et continue. Leur noyau est de forme ovale ou sphérique suivant l'incidence des coupes avec un nucléoplasme qui est soit homogène, soit avec une chromatine répartie en paquet à la périphérie. La matrice fondamentale du cytoplasme est faiblement contrastée, les mitochondries, peu nombreuses, sont très osmiophiles et le réticulum endoplasmique rugueux (RER), pourvu de nombreuses citernes, est très abondant dans les extensions cytoplasmiques et parfois aussi dans la région périnucléaire (fig. 5). Au stade de l'appari- 
tion de la lipogenèse, des inclusions lipidiques de taille restreinte et de forme irrégulière sont observées dans un nombre très réduit de ces cellules (fig. 6) comme cela est parfois observé darıs le cas de cellules du système nerveux (fig. 7) ou de cellules du type des plasmocyles.

- Les cellules de type endothélial, disposées auour des capillaires sanguins ef groupées en petits agrégats plus ou moins compacts, sont polymorphes. L'aspect ultrastructural de ces cellules est plus ou moins distinct suivant le niveau du prélèvement (fig. 8 et 9), suivant la couche d'un même niveau et parfois pour un même emplacement. Néanmoins, en tenant compte de l'évolution continue des cellules périvasculaires, on peut dire que pour chaque niveau étudié l'aspect ultrastructural de ces cellules est toujours comparable à celui des cellules, endothéliales des capillaires qu'elles entourent.

Observés au moment de l'apparition des premières inclusions lipidiques, les préadipocytes sans ou avec des lipides, d'aspect et de forme plus ou moins comparables, sont entourés d'une lame basale continue, parfois élargie ou entrecoupée. La membrane plasmique est régulière et généralement bien apparente ; elle est pourvue d'invaginations pinocytotiques variables en taille et en nombre sur le pourtour d'une même cellule.

Le noyau en position centrale, dans la majorité des cas, est oval ou sphérique suivant l'incidence des coupes et possède un, deux ou trois nucléoles. Le nucléoplasme est irrégulièrement contrasté sauf en de rares cas où il est homogène.

L'appareil de Golgi, les mitochondries et le réticulum endoplasmique apparaissent en quantité variable suivant le niveau du prélèvement et pour un même niveau suivant les cellules. Cependant, compte tenu du fait que la répartition des organites cellulaires varie d'une cellule à l'autre et suivant la région d'une même cellule, il s'avère difficile d'apprécier la quantité de ces organites.

Au stade débutant de la lipogenèse, les inclusions lipidiques sont observées, suivant les groupes, dans une ou plusieurs cellules. Cependant, en très peu de temps la plupart des cellules d'un même groupe contiennent des lipides en quantité comparable.

\section{PLANCHE II}

Observés en microscopie électronique, éléments du tissu adipeux de couverture chez le fœtus de porc à différents âges.

FIG. 5. - Vue partielle d'une coupe effectuée dans la couche exferne à l'emplacement du tissu adipeux de couverfure ou niveau du cou chez un fœfus âgé de 50 jours. Les cellules de fype fibroblastique, (incidence de coupes variées) riche en réticulum endoplasmique rugueux, RER, sont inégalement disséminées. $\nsucc 2500$.

FIG. 6. - Vue partielle d'une cellule de type fibroblastique de la couche externe au niveau du cou au stade d'apparition de la lipogenèse. Une inclusion lipidique, L, isolée, de forme irrégulière est très contrastée. Le réticulum endoplasmique rugueux, RER, est abondant et élargi. Mitochondries, $M$; noyau, $N$; membrane plasmique, MP. $\nsucc 48000$

FIG. 7. - Chez le même fołus ef au même emplacement que celui de la figure 6, inclusion lipidique dans une cellule du système nerveux. Membrane plasmique, MP ; lame basale, B. $\succ 33000$. 
PI. II
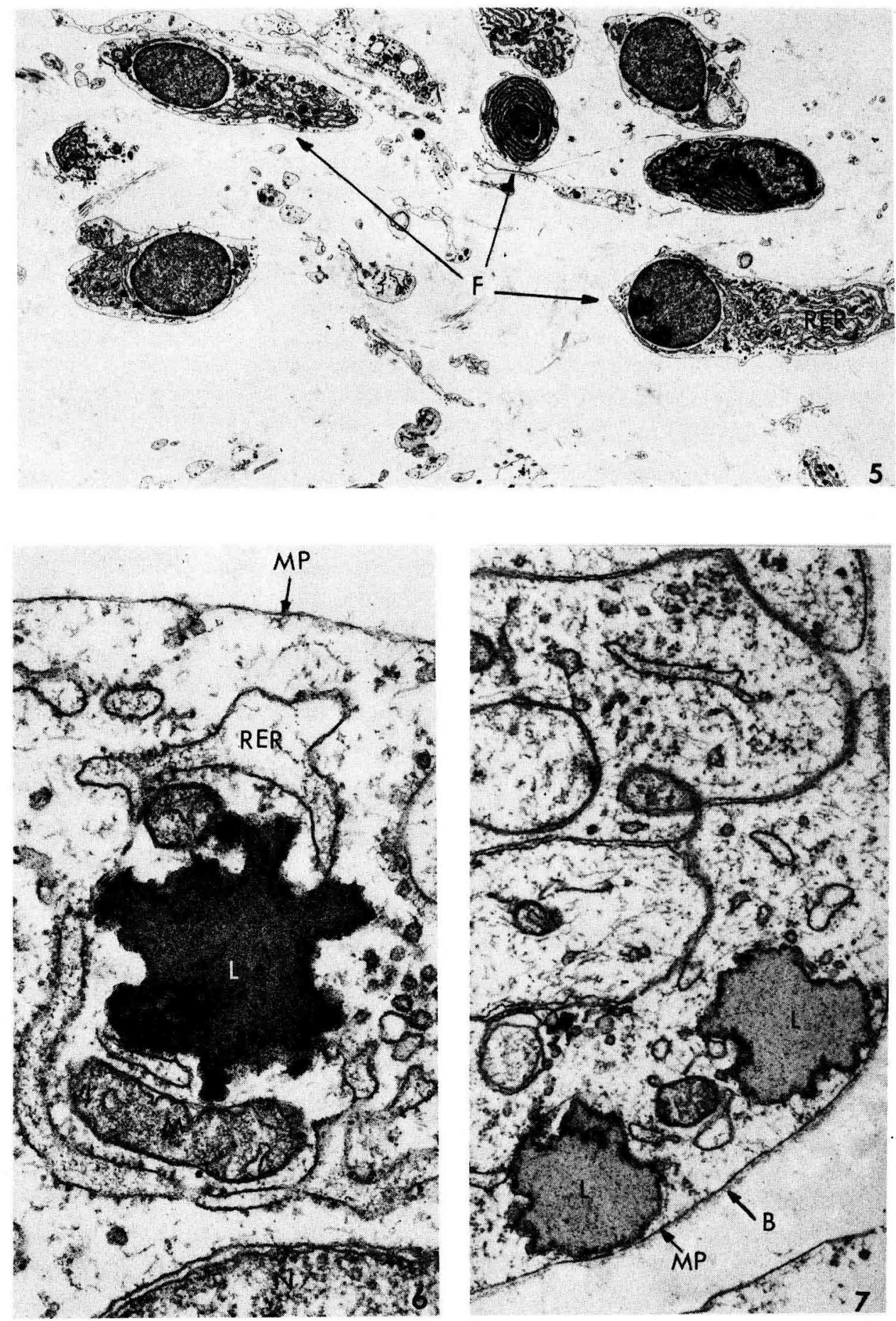
Simultanément avec l'extension de la lipogenèse, il y a apparition de nouveaux capillaires sanguins et prolifération à leur pourtour de nouvelles cellules qui à leur tour, accumulent des lipides.

Les premières inclusions lipidiques sont isolées ou en nombre variable ef leur localisation dans le cytoplasme ne semble pas être prédéterminée.

Dans un premier temps et pour la majorité des cellules adipeuses le nombre des inclusions lipidiques de volume variable s'accroît. Puis, du fait de l'augmentation de leur volume, ces inclusions se trouvent en contact et fusionnent. A partir de ce moment, le nombre des inclusions lipidiques par cellule est soit en augmentation, soit en diminution selon que l'apparition des nouvelles inclusions lipidiques est supérieure ou' inférieure au nombre des fusionnements.

D'autres cellules adipeuses, moins nombreuses, ont dès le début de la lipogenèse une seule inclusion lipidique importante généralement située assez près du noyau et en position centrale. Sur le pourtour de cette inclusion principale se forment d'autres inclusions de petite taille qui rapidement fusionnent avec l'inclusion centrale. De ce fait on observe, à tout moment, une inclusion volumineuse entourée de petites inclusions.

D'une façon générale ef cela pendant toute la durée de nos observations, c'està-dire chez le fœtus âgé de 52 à 80 jours, les cellules adipeuses sont toujours pourvues de plusieurs inclusions lipidiques ce qui veut dire qu'elles sont en évolution et qu'aucune cellule n'a encore atteint le stade adipocyte : « cellule univacuolaire».

Pour un groupe de cellules, à un niveau donné, ef si les sections passent par le centre des cellules, le diamètre cellulaire est comparable ce qui indique que les préadipocytes apparaissant en même temps se remplissent de lipides de façon uniforme. Les groupes de cellules qui apparaissent par la suite, toujours autour des capillaires en formation, soit à la périphérie du panicule, soit à d'autres emplacements, se remplissent de lipides à leur tour.

L'accroissement du volume cellulaire, dô à l'accumulation de lipides, fait que les cellules d'un même groupe se rapprochent ou viennent en contact les unes avec les autres. Cependant, il est difficile de savoir, en raison de la présence des nombreuses projections cytoplasmiques, si la surface cellulaire à ce stade est simplement due à

\section{PLANCHE III}

Observés en microscopie électronique, éléments du tissu adipeux de couverture chez le fœetus de porc à différents âges.

FIG. 8. - Aspect de quelques cellules adipeuses, CA, ef de préadipocytes sans lipides, P, groupés autour des vaisseaux sanguins, $\mathrm{C}$, prélevés chez un fœtus âgé de 78 jours dans la couche interne au niveau du cou. Les inclusions lipidiques, L, sont très nombreuses et moyennement constrastées. Le noyau, $N$, est situé à la périphérie des cellules et les organites cellulaires sont en majorité situés entre les inclusions lipidiques. $\times 3400$.

FIG. 9. - Chëz le même porcelet que celui de la figure 8 et dans la couche correspondante, aspect d'éléments de tissu adipeux prélevés au niveau du dos. Cellules adipeuses, $C A$; inclusions lipidiques, $L$; noyau, $\mathrm{N}$; vaisseaux sanguins, $C . \times 3400$. 

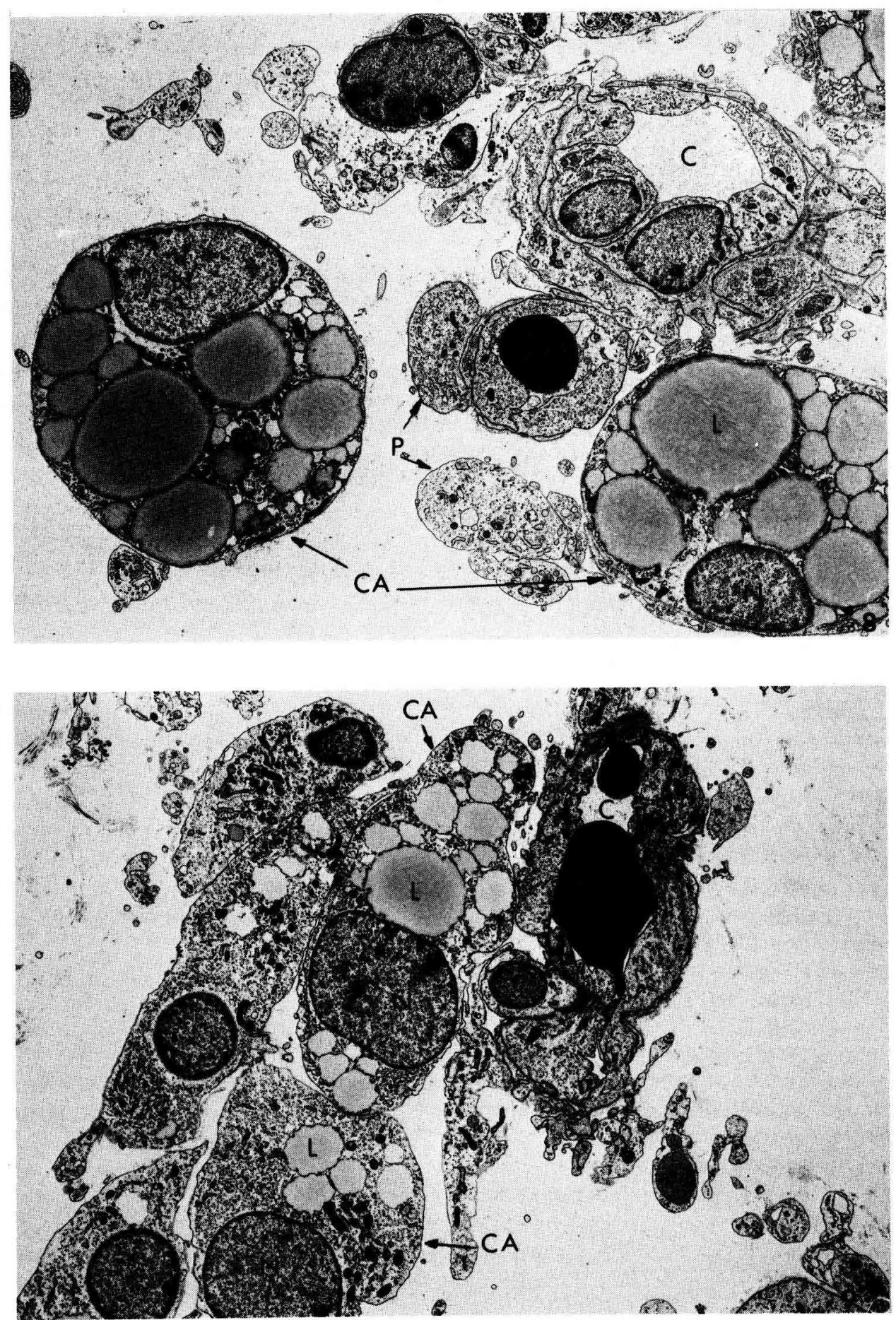
l'extension des projections cytoplasmiques ou à l'augmentation de la surface de la membrane plasmique.

Les inclusions lipidiques repoussent presque toujours le noyau vers la périphérie de la cellule ef les cas sont rares où il reste au centre de la cellule entouré des inclusions lipidiques. Les organites cytoplasmiques sont à ce stade repoussés vers la périphérie de la cellule, ils sont entourés par les inclusions lipidiques et progressivement dégradés.

\section{Discussion.}

\section{Apparition de la lipogenèse.}

Les présentes observations, effectuées sur des fœtus de Porc, de race Large-White (le régime maternel est normal et équilibré), montrent que l'apparition de la lipogenèse aux niveaux inguinal, dorsal, interscapulaire, du cou et du sternum est simultanée ou presque et qu'elle a lieu chez le fotus entre le $52^{\mathrm{e}}$ et le $56^{\mathrm{e}}$ jour de la gestation.

En attendant des renseignements complémentaires, on peut présumer que les niveaux des prélèvements sont représentatifs et que l'ensemble des tissus adipeux de couverture apparaît au même moment ou tout au plus à quelques jours d'intervalle. Ces observations nous indiquent également que le poids du fœtus, son emplacement dans la corne utérine ou le nombre de fœtus par portée ne semble pas modifier le moment d'apparition des cellules adipeuses. Le facteur nutrition, quant à lui, n'a pas encore été élucidé.

Si on se refère aux travaux concernant les tissus adipeux internes chez le fotus de Porc de la même race, on constate que l'apparition des tissus adipeux de couverture a lieu environ une semaine avant celle des tissus adipeux péricardiaque, épididymaire (Desnoyers et Vodovar, 1974) et périrénal (Desnoyers ef Vodovar, 1977) ef deux mois avant celle du tissu adipeux mésentérique (Vodovar, Desnoyers ef François, 1971).

Par rapport au Rat, cette précocité est encore beaucoup plus marquée puisque les tissus adipeux des emplacements correspondants apparaissent, chez cette dernière espèce, dans un temps très proche de celui de la naissance. Par contre, en tenant compte de la durée de la gestation, cette précocité serait comparable à ce qui a été observé pour l'Homme (Simon, 1962 ; Wassermann, 1965).

\section{Origine des cellules adipeuses.}

Mersmann, Goodman ef Brown (1975) indiquent que l'aspect des cellules des tissus adipeux sous-cutanés du porcelet après la naissance pourrait être assimilé à l'un des quatre types cellulaires suivants : cellules endothéliales, réticulaires, fibroblastiques et plasmocytaires.

Au stade actuel de nos observations, nous avons noté que les cellules dans lesquelles les inclusions lipidiques apparaissent sont, en grande majorité, situées autour des capillaires sanguins et du type des cellules endothéliales. D'autres cellules, peu nombreuses, de types fibroblastique ou plasmocytaire et des cellules du système nerveux contiennent également des inclusions lipidiques, généralement isolées et de 
petit volume. Néanmoins, en accord avec d'autres travaux (Simon, 1962) nous pensons que les cellules ayant ces derniers types cellulaires, tout en ayant la capacité d'accumuler des lipides en quantité limité à un moment donné, ne deviendront pas des adipocytes et que seules les cellules de type endothélial sont les précurseurs des cellules adipeuses.

Notre conviction concernant la provenance des préadipocytes des tissus adipeux de couverture est étayée par la ressemblance ultrastructurale existant entre les cellules endothéliales aux différents emplacements étudiés et les premières cellules adipeuses, par le mode de prolifération et la répartition des préadipocytes dans le tégument du fœetus et enfin par la comparaison avec nos études antérieures concernant les tissus adipeux internes (Vodovar, Desnoyers et François, 1971 ; Desnoyers et Vodovar, 1974 ; Desnoyers et Vodovar, 1977).

\section{Prolifération des préadipocytes et leur évolution.}

Peu de temps avant l'apparition de la lipogenèse ef par la suite pendant la durée de nos observations, nous avons pu noter que la prolifération cellulaire est surtout importante à la périphérie des capillaires sanguins alors que la population cellulaire disséminée et de type fibroblastique paraît conserver ce même aspect ef la même densité.

C'est aussi dans les cellules périvasculaires, dont l'apparition est liée de façon évidente à la formation des capillaires sanguins, qu'apparaissent et se développent les multiples inclusions lipidiques.

Les observations ultrastructurales concernant l'évolution des préadipocytes sans inclusion lipidique sont très réduites, malgré le prélèvement quotidien de tissu, car le temps entre la prolifération des préadipocytes et l'apparition des lipides dans leur cytoplasme semble être très court.

Par contre, l'évolution des cellules adipeuses au stade d'apparition de la lipogenèse chez le fotus de Porc est lente à ce niveau si on la compare au même stade à celle des cellules au niveau du mésentère chez la même espèce ou à celle du Rat.

\section{Aspect, taille ef nombre de cellules adipeuses.}

Comme nous l'avons exposé, et mises à part quelques rares cellules de provenance variable contenant très peu d'inclusions lipidiques, l'aspect ultrastructural des préadipocytes sans lipides ou avec les premières inclusions lipidiques est plus ou moins distinct selon le niveau du prélèvement. D'autre part, mis à part le degré d'évolution, l'aspect ultrastructural de ces cellules est dans tous les cas comparable à celui des cellules endothéliales des capillaires sanguins du même niveau. A la suite de ces deux observations, et jusqu'à la preuve du contraire, nous pensons que les différences observées entre l'ultrastructure des préadipocytes des différents niveaux proviendraient des différences existant entre l'ultrastructure des cellules endothéliales des capillaires sanguins de ces mêmes niveaux.

Nous avons indiqué que la taille des cellules adipeuses est variable suivant le niveau du prélèvement comme cela a déjà été montré pour les tissus adipeux internes de la même espèce (Vodovar, Desnoyers ef François, 1971 ; Desnoyers ef Vodovar, 1974 ; Desnoyers et Vodovar, 1977) et pour d'autres espèces (Nouguès, 1975 ; Desnoyers, 1977). 
La différence, nettement établie, des tailles cellulaires pour les cellules adipeuses des tissus des différents emplacements et qui apparaissent en même temps, n'est pas élucidé. Parmi les hypothèses possibles on peut penser que la vitesse de prolifération des préadipocytes n'est pas comparable aux différents niveaux tandis que l'afflux des lipides est le même ou inversement que l'afflux des lipides vers les différents niveaux est variable alors que la vitesse de prolifération, toute proportion gardée, est identique.

Le problème de la détermination du nombre des cellules adipeuses et des préadipocytes sans lipides auquel nous portons le plus grand intérêt à ces premiers siades de formation des tissus adipeux de couverture chez le Porc, sera exposé ultérieurement.

\section{Réunion Groupe Développement INRA/Production animales Thiverval-Grignon, 14-15 avril 1976.}

Remerciements. - Cette étude a été réalisée avec l'appui financier de l'INSERM (contrat 73.4.460.19) el de l'INRA (ATP « Tissu adipeux »).

\section{Références}

ALEXANDROWICZ St., 1949. The accumulation of fat in the subcutaneous tissue of pig embryos. Bull. int. Acad. polon. Sc. Left., cl. Sci. Math. Not. Ser. B : Sci. Nat., 2 (Zool.), 261-275.

DESNOYERS F., 1973. Etude morphologique des adipocytes ef leur évolution chez le porc ef chez le rat. Thèse Doct. Paris.

DESNOYERS F., 1977. Ełude morphologique chez le rat, du tissu adipeux périrénal au stade de sa formation. Ann. Biol. anim. Biochim. Biophys. (sous presse).

DESNOYERS F., VODOVAR N., 1974. Apparition, origine et évolution des tissus adipeux épididymaire et péricardiaque du fœtus de porc. Ann. Biol. anim. Biochim. Biophys., 14, 769-780.

DESNOYERS F., VODOVAR N., 1977. Etude structurale ef ultrastructurale du tissu adipeux périrénal au stade de sa formation chez le porc. Ann. Biol. anim. Biochim. Biophys., 17, 269-282.

FOWLER E. H., CALHOUN M. L., 1964. The microscopic anatomy of developing fetal pig skin. Am. J. vel. Res., 25, 156-164.

HERMANS P. G. C., 1973. The development of adipose tissue in swine foituses (A morphology study). Tijdschr. Diergeneesk., 98, 662-667.

KNITTLE J. L., HIRSCH J., 1968. Effect of early nutrition on the development of rat epididymal fat pads : cellularity and metabolism. J. clin. Invest., 47, 2091-2098.

LEE Y. B., KAUFFMAN R. G., GRUMMER R. M., 1973. Effect of early nutrition on the development of adipose tissue in the pig. I. Age constant basis. J. anim. Sci., 37, 1312-1325.

LEMONNIER D., 1972. Effect of age, sex and site on the cellularity of the adipose tissue in mice and rats rendered obese by a high fat diet. J. clin. Invest., 51, 2907-2915.

MERSMANN H. J., GOODMAN J. R., BROWN L. J., 1975. Development of swine adipose tissue : morphology and chemical composition. J. Lipid Res., 16, 269-279.

NOUGUĖS J., 1975. Adipocyte growth of four adipose deposits in rabbit. Ann. Biol. anim. Biochim. Biophys., 15, 541-546.

SIMON G., 1962. Genèse et structure du tissu adipeux chez l'Homme. Acta anat., 48, 232-241.

SMITH J. L., CALHOUN M. L., 1964. The microscopic anatomy of the integument of newborn swine. Am. J. vet. Res., 25, 165-173.

VODOVAR N., SERRES F., FRANÇOIS A. C., 1969. Aspect morphologique de la formation du dépôt adipeux mésentérique chez le porcelef. C. R. Acad. Sc. Paris, Série D, 269, 969-971.

VODOVAR N., DESNOYERS F., FRANÇOIS A. C., 1971. Origine et évolution des adipocytes mésentériques du porcelet avant la naissance. Aspect ultrastructural. J. Microsc., 11, 265-284.

VODOVAR N., DESNOYERS F., FLANZY J., 1972. Dépôt mésentérique du porcelet : étude morphologique. Ann. Biol. anim. Biochim. Biophys., 12, 243-262.

WASSERMANN F., 1965. The development of adipose tissue. In REYNOLD A. E., CAHILL G. F. Jr. Hanbook of physiology : adipose tissue, 5, 87-100. Am. Phys. Soc., Washington D. C. 